\title{
The WinBraille Approach to Producing Braille Quickly and Effectively
}

\author{
Mats Blomquist ${ }^{1}$ and Per Burman ${ }^{2}$ \\ ${ }^{1}$ Luleå University of Technology, 97187 Luleå, Sweden \\ Mats.Blomquistesm.luth.se \\ ${ }^{2}$ Index Braille AB, 95428 Gammelstad, Sweden \\ per.burmaneindexbraille.se
}

\begin{abstract}
With WinBraille it is possible to emboss directly from a word processor like Microsoft Word on any of Index Braille's embossers, and on-thefly contract the document into grade 2 Braille. The benefit of WinBraille is that it has a simple interface for the novice, but is still powerful for the advanced user. WinBraille contains Windows drivers for Index Braille's embossers, the WinBraille application, contraction rules for more than 20 languages, a contraction rule editor, predefined profiles and templates for immediate access, and macros to directly emboss from Microsoft Word. The combination of Word macros, drivers, predefined contraction rules, profiles and templates makes the system suitable for the user with no or limited knowledge of Braille and Braille printing. We describe how the WinBraille's approach can facilitate the daily work for people who normally do not print Braille, but occasionally have to produce materials to be read by blind or visually impaired people. We also explore some of WinBraile's less advanced features, like Braille formatting and how to select different profiles and templates.
\end{abstract}

\section{Introduction}

WinBraille is a Windows program for producing and embossing Braille on Index Braille's embossers. The WinBraille system contains the WinBraille program, printer drivers for Index Braille's embossers, contraction rules and macros for Microsoft Word.

A central concept is the profile. A WinBraille profile is a container for all WinBraille settings, like Braille contraction rules and Braille formatting and templates. WinBraille contains predefined profiles for a number of applications.

Contraction rules exist for the following languages: American English, Belgian, Brazilian Portuguese, Bulgarian, Czech, Danish, Dutch, English, French, German, Greek, Icelandic, Italian, Latvian, Nigerian, Norwegian, Polish, Portuguese, Russian, Sami, Slovak, Spanish, Swedish, and Turkish. 


\section{Embossing a Document}

There is no need to specifically know how to format a Braille document for a specific purpose, or translate the context to contracted Braille. The predefined profiles contain all the necessary information. Embossing a document with WinBraille is as easy as printing the document from Word to the standard printer. The embossing process is described in the following figure:
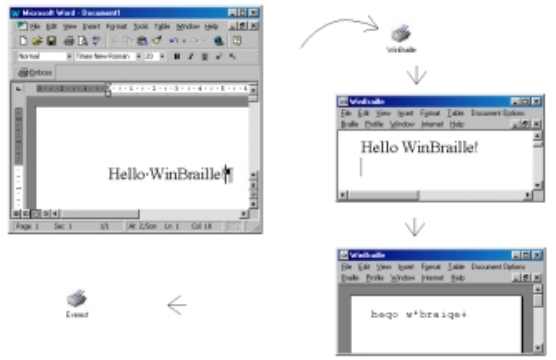

Fig. 1. The embossing Process

When we emboss from Word with the WinBraille's Word macros, an emboss dialog, similar to the standard print dialog, will appear:

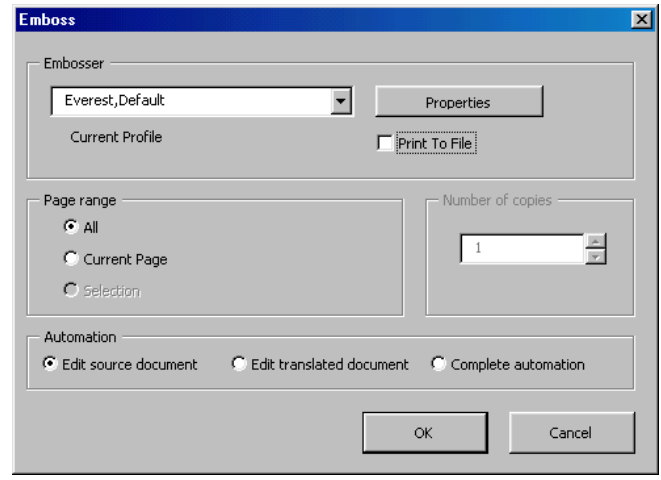

Fig. 2. Emboss Dialog

The emboss dialog gives us an option to select a proper profile. The "Complete automation" option lets us emboss to the selected embosser, using predefined settings from the selected profile.

\section{References}

1. Mats Blomquist, WinBraille Contraction Rules and Rule File Editor, available on Index Braille's home page, http://www.indexbraille.com, May 2002.

2. Index Braille's home page, http://www.indexbraille.com, visited January 2002. 